from: The Myositis Association, I. Targoff Consultant for: Oklahoma Medical Research Foundation Clinical Immunology Laboratory regarding myositis autoantibody testing, F. Miller: None declared, L. Rider: None declared, A. Mammen: None declared

DOI: 10.1136/annrheumdis-2017-eular.6246

\section{SAT0377 RELIABILITY OF A NEW AUTOMATED SYSTEM FOR ABSOLUTE CAPILLARY NUMBER COUNTING (AUTOCAPI) ON SYSTEMIC SCLEROSIS NAILFOLD VIDEOCAPILLAROSCOPIC IMAGES}

M. Cutolo ${ }^{1}$, K. Melsens ${ }^{2}$, A.C. Trombetta ${ }^{1}$, C. Pizzorni ${ }^{1}$, E. Deschepper ${ }^{3}$, A. Sulli ${ }^{1}$, V. Smith ${ }^{4} .{ }^{1}$ Research Laboratory and Academic Division of Clinical Rheumatology, Department of Internal Medicine, University of Genoa, Genoa, Italy; ${ }^{2}$ Department of Internal Medicine, Faculty of Medicine and Health Sciences: ${ }^{3}$ Department of Public Health, Biostatistics Unit; ${ }^{4}$ Department of Internal Medicine, Faculty of Medicine and Health Sciences; Department of Rheumatology, Ghent University Hospital, Ghent University, Ghent, Belgium

Background: Nailfold capillary density is a useful measure in systemic sclerosis (SSc) classification and evaluation. Its manual detection may be time-consuming, hampering its use in largescale investigations. We evaluated a new automated system to assess the absolute nailfold capillary number.

Objectives: To attest the instrumental reliability of automatic counting in SSc patients using nailfold video capillaroscopy (NVC) images.

Methods: 75 NVC random images, from SSc patients, were blindly analyzed by four raters (2 less and 2 more experienced; raters: 1,2,3,4) from two European centers. Each rater was asked to define the region of interest (ROI) on the NVC images and to manually count the number of capillaries, according to the following instructions: upper bound placed on top of the longest capillary head and lower bound placed on half of the length of that longest capillary (figure 1); if the common branch of an abnormal shape (neoangiogenesis) is in ROI it is counted as being one; if the common branch is out of $\mathrm{ROI}$ it is counted as separate capillaries; if the capillary is on the edge of the vertical line of ROI, it is only counted when the head of the capillary is half in ROI; if the capillary head is on the edge of lower bound, it is counted as soon as the "head" part is in the ROI; all "heads" in the ROI are counted (not only distal row). The dedicated automated system (AUTOCAPI-ds medica, IT) also counted the number of capillaries in the same ROI (figure 1). Reliability between the manual and automatic counting was investigated per rater through intraclass correlation coefficient (ICC) and reported with $95 \%$ confidence interval $(\mathrm{Cl})$. External validation was obtained by multi-rating of the same set of images. Average difference between automated and manual counting per rater was calculated.

Results: The ICC $(95 \% \mathrm{Cl})$ of manual versus automatic counting in $\mathrm{ROI}$ was 0.77 $(0.61-0.86)$ for rater $1(p<0.0001), 0.81(0.71-0.88)$ for rater $2(p<0.0001), 0.65$ $(0.50-0.76)$ for rater $3(\mathrm{p}<0.0001)$ and $0.81(0.71-0.87)$ for rater $4(\mathrm{p}<0.0001)$. The average difference was -0.69 for rater $1,0.04$ for rater $2,-0.03$ for rater 3 and 0.16 for rater 4

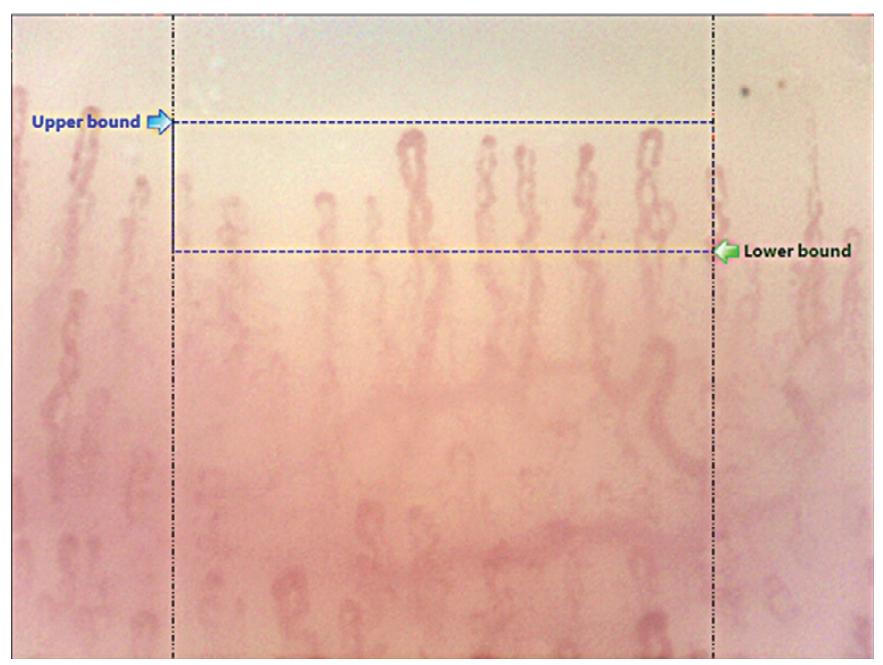

Figure 1. In the picture captured by the operator (normal subject), two vertical bars, with a distance of $1 \mathrm{~mm}$ appears automatically in the middle of the picture, together with two horizontal bars (upper bound and lower bound) to be placed manually (see text) in order to define the ROI.

Conclusions: This is a first study to attest the reliability of a new automated system to calculate the absolute number of capillaries in a ROI arising from SSc NVC images. High performance of the new automated counting system was confirmed in pathological conditions (SSc).

Disclosure of Interest: None declared

DOI: 10.1136/annrheumdis-2017-eular.3191

\section{SAT0378 BONE MARROW OEDEMA AND SYNOVITIS ON MRI OF THE HAND ARE ASSOCIATE WITH DIGITAL ULCERS, ACTIVE DISEASE AND IMPAIRED FUNCTIONAL CAPACITY IN SYSTEMIC SCLEROSIS}

B. Stamenkovic ${ }^{1,2}$, S. Stojanovic ${ }^{1,2}$, J. Nedovic ${ }^{2}$, V. Zivkovic ${ }^{1,2}$, S. Milenkovic ${ }^{2}$, J. Jovanovic ${ }^{2}$, N. Damjanov ${ }^{3}$, A. Stankovic ${ }^{1,2} .{ }^{1}$ Medical School; ${ }^{2}$ Rheumatology, Institute Niska Banja, Nis; ${ }^{3}$ Rheumatology, Institute for Rheumatology, Belgrade, Serbia

Background: Hand inflammatory involvement is a major feature in patients with systemic sclerosis (SSc), responsible for major disability. Magnetic resonance imaging (MRI) can identify and characterize subclinical inflammation and joint damage on hand with much greater precision than clinical, radiographic and ultrasonography assessment in SSc.

Objectives: To determine the association of MR bone marrow oedema, synovitis and probability for the occurrence of listed inflammatory changes with clinical features and laboratory tests in SSc patients

Methods: 112 SSc patients were tested (mean age 54y). Contrast enhanced low field MRI of the wrist and MCP2-5 joints was performed to all the pts. MRI bone marrow oedema and synovitis were assessed and scored by OMERACT RAMRIS scoring system. Age, sex, SSc type, disease duration (date of first non Raynaud symptom), Raynaud phenomenon, articular or periarticular pain, joint swelling and contractures, digital ulceration, $\mathrm{HAQ}$, acroosteolysis (by standard PA radiographs of hand and wrist) pulmonary arterial hypertension (PAP $>40 \mathrm{mmHg}$ at rest on Doppler echocardiography), pulmonary fibrosis (by CT and pulmonary function tests) and laboratory tests (antibody profile, RF, CRP, Creatine phosphokinase) and disease activity (by Valentini index) were done.

Results: By multiple logistic regression analysis taking into account all clinical and laboratory variable, we found that MRI bone marrow oedema of the hand was associated and probability for the occurance of MRI bone marrow oedema was higher for the SSc pts with digital ulcers (OR=6.081;95\%IP:1.295-28.550; $\mathrm{p}<0.05), \quad H A Q>1.5(\mathrm{OR}=6.448 ; 95 \% \mathrm{IP}: 1.714-24.257 ; \mathrm{p}<0.01)$ and active disease (OR=3.377; 95\%IP; 1.175-9.706; $p<0.05)$

MRI synovitis of the hand was associated and probability for the occurance of MRI synovitis was higher, also, for the SSc pts with digital ulcers (OR=5.128; 95\%IP: 1.085-24.243; $p<0.05$ ), HAQ>1.5 (OR=9.067; 95\%IP: 1.925-42.708; $p<0.01)$ and active disease (OR=3.565; 95\%IP: $1.181-10.764 ; \mathrm{p}<0.05)$.

Conclusions: MRI bone marrow oedema and synovitis on the hand in SSc are associate with digital ulcers, impaired functional capacity and active disease. Monitoring and treatment of clinical features and organ involvement are essential in all the pts with SSc, especially those with proven bone marrow oedema and synovitis on MRI of the hand.

Disclosure of Interest: None declared

DOI: 10.1136/annrheumdis-2017-eular.4522

\section{SAT0379 NAILFOLD CAPILLAROSCOPY FINDINGS IN PATIENTS WITH INFLAMMATORY MYOPATHY AND/OR SPECIFIC OR ASSOCIATED ANTIBODIES}

A.M. Millan Arciniegas ${ }^{1}$, M.A. Martinez ${ }^{2}$, A. Baucells ${ }^{2}$, C. Juarez ${ }^{2}$, L. Martinez ${ }^{2}$, H.S. Park ${ }^{1}$, B. Magallares ${ }^{1}$, A. Laiz ${ }^{1}$, P. Moya ${ }^{1}$, J.M. Llobet ${ }^{1}$, C. Diaz Torne ${ }^{1}$, I. Castellvi ${ }^{1} .{ }^{1}$ Rheumatology; ${ }^{2}$ Immunology, Hospital de Sant Pau, Barcelona, Spain

Background: Nailfold videocapillaroscopy (NVC) is an easy, fast and nonaggressive tool, useful in the study of autoimmune diseases. The use of NVC in Inflammatory Myopathy (IM) is not clearly established.

Objectives: 1 . To evaluate capillaroscopic findings in patients with IM and/or with presence of specific or associated antibodies with this pathology. 2. To analyze possible relationships with clinical characteristics of the patients.

Methods: Retrospective review of a cohort of patients with IM and/or with presence of specific or associated antibodies, followed in Rheumatology Unit of a University Hospital.

Patients underwent a NVC at 200x, being evaluated for the presence of: loss of capillary density, enlarged and giant capillaries, ramified capillaries, haemorrhages, thrombosis, tortuous capillaries, avascular areas, disorganization of capillary architecture and subpapilar venous plexus. The following variables were also collected: sex, age, active smoking, muscle weakness, CK elevation at diagnosis, compatible muscle EMG and biopsy, skin findings, cardiac disease, dysphagia, lung disease, Raynaud's phenomenon, cancer history and overlap syndromes.

For the comparison of qualitative and/or quantitative variables Fisher's exact Test or T-test was performed when necessary.

Results: Twenty patients with at least one NVC (45\% with 2), $65 \%$ female, with a mean age of 58 years \pm 11.6 were evaluated. The characteristics of the patients are detailed in table 1 .

$65 \%$ of patients had some capillaroscopic alteration. The findings in NVC-1 and NVC-2 were: loss of capillary density $30 \%$ and $33 \%$, tortuous capillaries $90 \%$ and $89 \%$, enlarged capillaries $65 \%$ and $66.7 \%$ (giants $30 \%$ and $33 \%$ ), ramifications $40 \%$ and $55.6 \%$, disorganization $10 \%$ and $33 \%$, haemorrhages $25 \%$ and $44 \%$, thrombosis $20 \%$ and $0 \%$, avascular areas $25 \%$ and $22 \%$, visible venous plexus $40 \%$ and $55 \%$.

The presence of dysphagia was associated with the presence of loss of capillary 